S. P. MILLER*, W. J. MORRIS*, R. K. ORR, J. ECKERT, J. MILAN, M. MAUST, C. COWDEN,

J. CUI (MERCK \& CO., RAHWAY, USA)

Synthesis of the $\gamma$-Secretase Modulator MK-8428

J. Org. Chem. 2017, 82, 2957-2964.
Gategory

Synthesis of Natural

Products and

Potential Drugs

\section{Synthesis of MK-8248}<smiles>O=C(O)C(=O)c1cc(F)c(F)c(F)c1</smiles>

A

$\mathrm{mp} 164^{\circ} \mathrm{C}$

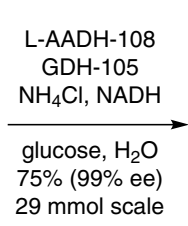

$29 \mathrm{mmol}$ scale

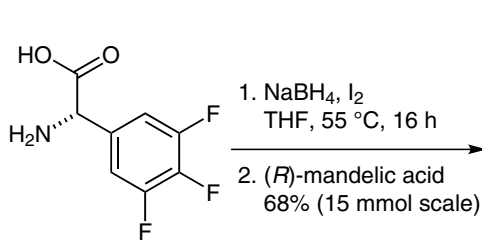

B
$\bullet(R)$-mandelic acid

$\mathrm{H}_{2} \mathrm{~N}^{\prime \prime \prime}$<smiles>C[C@H](CO)c1cc(F)c(F)c(F)c1</smiles>

C

$\mathrm{mp} 201^{\circ} \mathrm{C}$

$\mathrm{mp} 163^{\circ} \mathrm{C}$

\section{Key words}

\section{MK-8248}

$\gamma$-secretase

modulator

amino acid

dehydrogenase ring formation

reductive amination

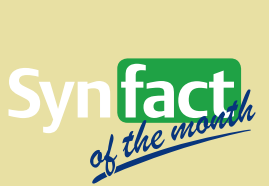

1,2,4-oxadiazine<smiles>COc1cc(/C=C(\CCCCl)C(=O)O)ccc1-n1cnc(C)c1</smiles>
equiv) EDC.HCl (7.0 equiv) $83 \%$ (9.62 mmol scale)<smiles>COc1cc(/C=C(\CCCCl)C(=O)N[C@H](CO)c2cc(F)c(F)c(F)c2)ccc1-n1cnc(C)c1</smiles>

$\mathrm{NaOMe}$ (1.5 equiv) THF, r.t., $2 \mathrm{~h}$ $97 \%(6.9 \mathrm{mmol}$ scale $)$<smiles>COc1cc(/C=C2\CCCN([C@@H](CON)c3ccccc3)C2=O)ccc1-n1cnc(C)c1</smiles>

H $\mathrm{HO}-\mathrm{NH}_{2}$
$25{ }^{\circ} \mathrm{C}, 2 \mathrm{~h}$ $\mathrm{H}_{2} \mathrm{O}, 25^{\circ} \mathrm{C}, 2 \mathrm{~h}$ $86 \%$ from $\mathbf{F}$<smiles>COc1cc(/C=C2\CCCN([C@@H](CON)c3cc(F)c(F)c(F)c3)C2=O)ccc1-n1cnc(C)c1</smiles>

$\mathrm{MsCl}$ (1.5 equiv) $\mathrm{Et}_{3} \mathrm{~N}$ (6.0 equiv) THF, -25 to $-15^{\circ} \mathrm{C}$

\section{2. $\mathbf{G}$ (1.5 equiv)} $25^{\circ} \mathrm{C}, 18 \mathrm{~h}$ $6.4 \mathrm{mmol}$ scale<smiles>O=C1c2ccccc2C(=O)N1O</smiles><smiles>COc1cc(/C=C2\CCCN([C@H](CO)c3cc(F)c(F)c(F)c3)C2=O)ccc1-n1cnc(C)c1</smiles>
$\mathrm{mp} 158^{\circ} \mathrm{C}$
Significance: MK-8428 is a $\gamma$-secretase modulator that is of interest for the treatment of Alzheimer's disease. Key steps in the synthesis depicted are (1) an amino acid dehydrogenase mediated conversion of $\alpha$-keto carboxylic acid $\mathbf{A}$ into 3,4,5trifluoro-(S)-phenylglycine (B) and (2) a four-step sequence including a dehydrative intramolecular cyclization to form the oxadiazine ring.
Comment: On treating a solution of I with hexamethyldisilazane (HMDS) and catalytic amounts of trimethylsilyl trifluormethanesulfonate (TMSOTf), the desired intramolecular cyclization took place in high yield. This silyl-mediated dehydration provided a milder alternative to Brønsted acids. The target molecule MK-8428 was isolated as its crystalline hemi-fumarate salt.

SYNFACTS Contributors: Philip Kocienski

Synfacts 2017, 13(06), 0563 Published online: 16.05.2017

Dol: 10.1055/s-0036-1590388; Reg-No.: K02017SF 